\title{
The association between ambient fine particulate air pollution and physical activity: a cohort study of university students living in Beijing
}

Hongjun $\mathrm{Yu}^{1 *} \mathbb{B}$, Miao $\mathrm{Yu}^{2}$, Shelby Paige Gordon ${ }^{3}$ and Ruiling Zhang ${ }^{3}$

\begin{abstract}
Background: Air pollution has become a substantial environmental issue affecting human health and healthrelated behavior in China. Physical activity is widely accepted as a method to promote health and well-being and is potentially influenced by air pollution. Previous population-based studies have focused on the impact of air pollution on physical activity in the U.S. using a cross-sectional survey method; however, few have examined the impact on middle income countries such as China using follow-up data. The purpose of this study is to examine the impact of ambient fine particulate matter $\left(\mathrm{PM}_{2.5}\right)$ air pollution on physical activity among freshmen students living in Beijing by use of follow-up data.
\end{abstract}

Methods: We conducted 4 follow-up health surveys on 3445 freshmen students from Tsinghua University from 2012 to 2013 and 2480 freshmen completed all 4 surveys. Linear individual fixed-effect regressions were performed based on repeated-measure physical activity-related health behaviors and ambient $\mathrm{PM}_{2.5}$ concentrations among the follow-up participants.

Results: An increase in ambient PM 2.5 concentration by one standard deviation $\left(44.72 \mu \mathrm{g} / \mathrm{m}^{3}\right)$ was associated with a reduction in 22.32 weekly minutes of vigorous physical activity (95\% confidence interval $[\mathrm{Cl}]=24.88-19.77)$, a reduction in 10.63 weekly minutes of moderate physical activity $(95 \% \mathrm{Cl}=14.61-6.64)$, a reduction in 32.45 weekly minutes of moderate to vigorous physical activity (MVPA) $(95 \% \mathrm{Cl}=37.63-27.28)$, and a reduction in 226.14 weekly physical activity MET-minute scores $(95 \% \mathrm{Cl}=256.06-196.21)$. The impact of ambient $\mathrm{PM}_{2.5}$ concentration on weekly total minutes of moderate physical activity tended to be greater among males than among females.

Conclusions: Ambient $\mathrm{PM}_{2.5}$ air pollution significantly discouraged physical activity among Chinese freshmen students living in Beijing. Future studies are warranted to replicate study findings in other Chinese cities and universities, and policy interventions are urgently needed to reduce air pollution levels in China.

Keywords: Air pollution, Fine particulate matter, Physical activity, Moderate-to-vigorous physical activity, youth

\footnotetext{
*Correspondence: yuhj12@mail.tsinghua.edu.cn

'Department of Physical Education, Tsinghua University, Tsinghua Yuan Str, Beijing 100084, China

Full list of author information is available at the end of the article
} 


\section{Background}

Air pollution is a major environmental issue affecting human health worldwide. According to the WHO, ambient air pollution may have contributed to approximately 4.2 million deaths worldwide in 2015 [1]. One type of ambient air pollution that has received increasing attention is fine particulate matter, specifically $\mathrm{PM}_{2.5}$. Particulate matter (PM) is mainly derived from industrial activities such as power generation, which is the combustion of coal and fossil fuels produced when heated [2]. PM is a mixture of solid and/or liquid particles suspended in the air. $\mathrm{PM}_{2.5}$ refers to particles that are less than $2.5 \mu \mathrm{m}$ micrometers in diameter. $\mathrm{PM}_{2.5}$ can be effectively inhaled and deposited in the airway and alveolar surfaces, causing health problems [3]. Previous studies have shown that adverse health effects of short- and longterm exposure to $\mathrm{PM}_{2.5}$ include blood pressure, myocardial infarction, stroke and all-cause mortality [4-7].

Physical inactivity is a leading risk factor of noncommunicable disease (NCD). The WHO reports that it is the fourth leading cause of global mortality and leads to 3.2 million deaths each year [8]. Regular physical activity has many health benefits, including reduced allcause mortality and reduced chronic disease such as cardiovascular diseases, diabetes, colon and breast cancer, hypertension, obesity and depression [9]. The WHO recommends that adults participate in at least $150 \mathrm{~min}$ per week of physical activity at a moderate-to-vigorous (MVPA) intensity [10]. MVPA has significant health benefits for youth and adults, including preventing obesity and decreasing blood pressure [11-13]. The U.S. Department of Health and Human Services recommends that youth accumulate $\geq 60 \mathrm{~min}$ per day of MVPA to prevent obesity and to benefit their physical fitness, bone health, metabolic and cardiovascular health [14]. However, it is estimated that $23 \%$ of adults and a vast majority of adolescents and youth fall short on the recommended guidelines for MVPA per week [15-18].

Although the adverse effects of air pollution and physical inactivity on health risks have been well studied, little knowledge is known regarding air pollution impact on physical activity. Air pollution may dismiss people from engaging in regular physical activity through several mechanisms. Air pollution has been associated with respiratory diseases, such as asthma and bronchitis $[2,19]$, resulting in impaired exercise performance [20]. The presence of smog can also restrict people from engaging in outdoor physical activity [21]. Media alerts of air quality to inform the public about harmful air pollution may affect people's physical activity behavior [22]. A few studies have linked air pollution to a decrease in exercise performance in athletes [23, 24], but little previous research has reported the relationship between air pollution and physical activity based on population-based evidence. To our knowledge, a total of 4 population-based studies have estimated the relationship between air pollution and physical activity [21, 25-27]. Previous studies have mainly analyzed survey data from the Behavioral Risk Factor Surveillance System (BRFSS), a large cross-sectional survey of U.S. adults 18 years of age and older $[21,25,26]$. Air pollution contribution $\left(\mathrm{PM}_{2.5}\right)$ was consistently found to be associated with a modest, but measurable, decrease in individuals' leisure-time physical activity [21, 25, 26].

Despite the aforementioned work, two major gaps in the scientific literature remain. Previous studies have exclusively focused on the impact of air pollution on physical activity in the U.S., but few studies have examined middle-income countries such as China. In the U.S., most counties have already met the national air quality standards levels. There are less than 10 counties in the U.S. that do not meet the new national air quality standard in reaching $12.0 \mu \mathrm{g} / \mathrm{m}^{3}$ by 2020 [28]. Since starting in the 1980's, China has experienced rapid industrial and urban development. As a result, air pollution has become a substantial threat to public health in China, the largest middle-income country in the world. In 2010, air pollution in China was the fourth cause of mortality and led to 1.2 million premature deaths, almost $40 \%$ of the global total [29]. Beijing, the capital city of China, has been experienced severe air pollution, with the highest annual average level of $\mathrm{PM}_{2.5}$ exceeding $600 \mu \mathrm{g} / \mathrm{m}^{3}$ [3]. Yet, no similar relationship between air pollution and physical activity has been explored among young adults. Previous studies have analyzed cross-sectional data that was subject to confounding bias due to unobserved differences in individual characteristics. Behavioral change in response to temporal variations in air quality could not be assessed due to data unavailability. The only other middle-income country-based study available examined the influence of $\mathrm{PM}_{2.5}$ air pollution on health behaviors among university retirees in China [30]. High $\mathrm{PM}_{2.5}$ concentration was found to discourage retired older adults from engaging in daily physical activities [30]. The strength of this study was in its prospective design; however, this was limited by convenience sampling which comprised its external validity [27]. There are currently no follow-up large sample studies on examined the influence of $\mathrm{PM}_{2.5}$ air pollution on physical activity among youth populations.

The purpose of this study is to examine the follow-up relationship between ambient $\mathrm{PM}_{2.5}$ air pollution and behavioral outcomes pertaining to weekly physical activity levels among university freshmen in Beijing. We hypothesized that in response to elevated air pollution, study participants reduced their physical activity. 


\section{Methods}

\section{Participants}

A paper-based health survey was conducted on a regular basis during students' freshman year at Tsinghua University. The survey was administered in class by faculty and all freshmen participants took the survey within a oneweek health education class window (every other day, Mon to Fri). Survey participation was voluntary. Upon signing the consent form, survey participants were asked to complete a paper-based questionnaire and hand it in after the course. The survey included questions regarding one's sociodemographic, physical and mental health status, and health and risk behavior. The same survey was administered four times within the freshman year of newly admitted undergraduate students entering the university in 2012 (September 24-28, 2012; November 5-9, 2012; March 5-9, 2013; May 13-17, 2013). Survey participants were asked to report their student identification number and that information was used to link multiple survey questionnaires completed by the same respondent.

Figure 1 presents the analytic sample selection flowchart for the 4 follow-up surveys administered. A total of 3445 Tsinghua University freshmen enrolled in 2012 responded to the survey. Among them, 3343 participated in more than one survey, 3072 participated in 3 or more surveys and 2480 participated in all 4 surveys. Among those repeated respondents, 3223 to 3242 (dependent upon specific outcome variables) had non-missing values for the specific outcome and all covariates, therefore, were included in the regression analyses. The study was approved by the Tsinghua University Institutional Review Board (IRB\#2012534001).

\section{Environmental measures}

Environmental measures included average ambient $\mathrm{PM}_{2.5}$ concentration $\left(\mu \mathrm{g} / \mathrm{m}^{3}\right)$, average daytime temperature $(\mathrm{C})$, average wind speed $(\mathrm{m} / \mathrm{s})$ and percentage of rainy days in Beijing, China over the last seven days before the health survey was administered. Hourly ambient $\mathrm{PM}_{2.5}$ concentration data came from the Mission China Air Quality Monitoring Program run by the U.S. Department of State (September 17th 00:00 - 27th 23:00, 2012; October 29th 00:00 - November 8th 23:00, 2012; February 25th 00:00 March 8th 23:00, 2013; May 6th 00:00 - 16th 23:00, 2013). Daily weather data including daytime temperature, wind speed and precipitation came from the China Meteorological Administration.

To facilitate result interpretation, we standardized average ambient $\mathrm{PM}_{2.5}$ concentration over the last seven days through centering (i.e., subtracting the mean from each value) and then dividing by its own standard deviation (i.e., $\mathrm{PM}_{2.5} \mathrm{z}$-scores). The estimated coefficient of ambient $\mathrm{PM}_{2.5}$ concentration can thus be interpreted as the change of an outcome variable (e.g., total minutes of walking in the last week) with respect to a change of ambient $\mathrm{PM}_{2.5}$ concentration by one standard deviation $\left(44.72 \mu \mathrm{g} / \mathrm{m}^{3}\right)$.

\section{Physical activity measurements}

The short version of the International Physical Activity Questionnaire (IPAQ) was used to measure physical activity (PA). The short version (9 items) of the IPAQ has been validated in China [31-33]. Data from the short IPAQ provided information on the time spent walking and on moderate- and vigorous-intensity activities.

Total minutes of vigorous physical activity in the last week were constructed based on the answers to two questions adapted from the International Physical

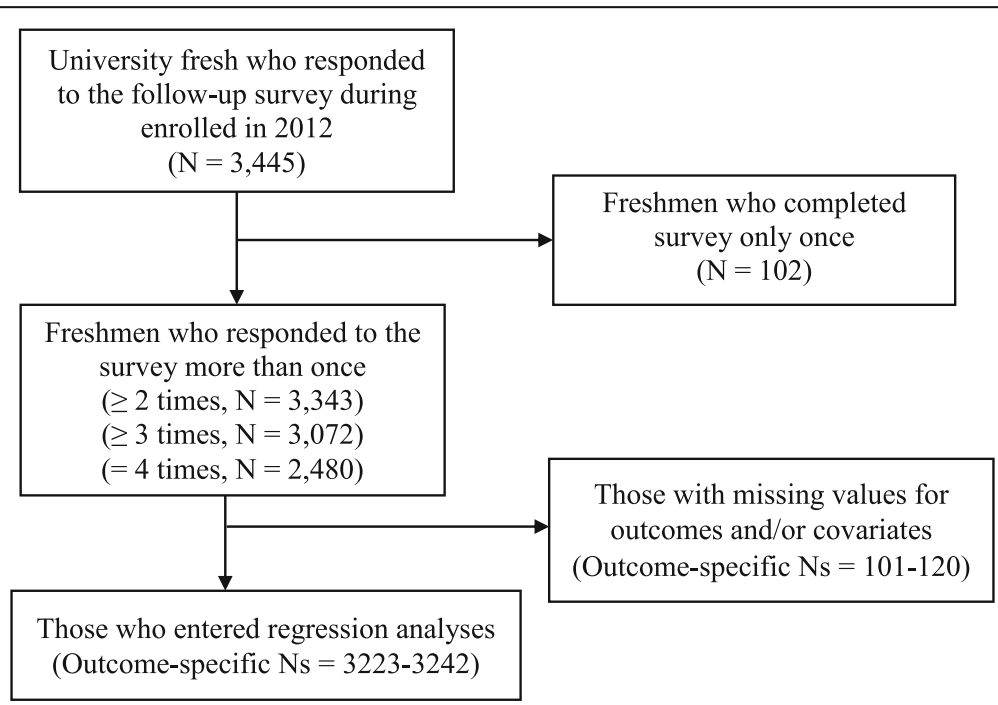

Fig. 1 Study Sample Flowchart 
Activity Questionnaire (IPAQ) [34]: "During the last seven days, on how many days did you do vigorous physical activities such as aerobics, running, fast bicycling and fast swimming?" and "How much time did you usually spend doing vigorous physical activities on one of those days?". Total minutes of vigorous physical activity in the last week were calculated through multiplying daily average number of minutes spent on vigorous physical activity by its corresponding number of days.

Total minutes of moderate physical activity in the last week were constructed based on the answers to two questions adapted from the International Physical Activity Questionnaire (IPAQ) [34]: "During the last seven days, on how many days did you do moderate physical activities, such as carrying light loads, bicycling at a regular pace and playing doubles tennis?" and "How much time did you usually spend doing moderate physical activities on one of those days?". Total minutes of moderate physical activity in the last week were calculated through multiplying daily average number of minutes spent on vigorous physical activity by its corresponding number of days.

Total minutes of walking in the last week were constructed based on the answers to the two questions adapted from the IPAQ [34]: "During the last seven days, on how many days did you walk for at least 10 minutes at a time?" and "How many minutes did you usually spend on one of those days walking?". Total minutes of walking in the last week were calculated through multiplying daily average minutes spent walking by its corresponding number of days.

Total weekly minutes of MVPA were calculated by weekly average of minutes spent on moderate physical activity plus weekly average of minutes spent on vigorous physical activity. Data collected using the IPAQ were used to estimate weekly total physical activity in metabolic equivalent (MET) energy expenditure. A METminute score is computed by multiplying the MET score by the minutes performed [35].

\section{Statistical methods}

Descriptive statistics including means, standard deviations and percentages were used to summarize and compare characteristics of the overall sample by sex. Chi-square tests were conducted to compare categorical variables and t-tests for continuous variables. These were repeat measures one-way ANOVA with Bonferoni(B) tests were conducted to compare the differences between the 4 follow-up surveys. Linear individual fixed-effect regressions were performed based on the repeated-measure survey data from freshmen student cohorts enrolled at Tsinghua University in 2012. The five continuous outcome variables were total minutes of vigorous physical activity, total minutes of moderate physical activity, total minutes of MVPA, total minutes of walking and total physical activity (MET-minute scores) in the week before the survey was administrated. The key independent variable in all regressions was the standardized average ambient $\mathrm{PM}_{2.5}$ air pollution concentration during the seven days prior to the survey. All models were controlled for the aforementioned individuallevel time-variant covariates as well as the environmental measures: average daytime temperature, average wind speed and percentage of rainy days in the last seven days. Separate regressions were conducted for each outcome variable based on samples stratified by sex (i.e., entire sample with both gender, male only and female only).

Compared to the conventional pooled cross-sectional regression, individual fixed-effect regression is preferred. Individual fixed-effect regression only uses withinindividual variations in physical activity level to identify the impacts of air pollution concentration, thus removing potential omitted variable bias due to differences in time-invariant individual characteristics such as genes, sex, ethnicity, habits and personal preferences. Due to the exclusive dependence upon within-individual variations in an outcome measure, individual fixed-effect regressions could only estimate the effect of a time-variant independent variable. Thus, time-invariant individual characteristics such as sex and ethnicity were not examined.

All statistical procedures were performed in Stata 14.2 SE version (StataCorp, College Station, TX). EickerHuber-White sandwich estimator was used to estimate the standard errors of regression coefficients, which addressed within-individual serial correlations.

\section{Individual-level covariates}

The following individual-level time-variant covariates were controlled for in the regression analyses: a continuous variable for age in years, a continuous variable for body mass index (BMI; $\mathrm{kg} / \mathrm{m}^{2}$ ) calculated from selfreported height and weight, a dichotomous variable for current smoking status (current non-smokers as the reference group), a dichotomous variable for current drinking status (current non-drinkers as the reference group), a continuous variable for self-rated physical health (on a scale of 1 to 10, worst to best) and a continuous variable for self-rated mental health (on a scale of 1 to 10 , poor to excellent).

\section{Results}

\section{The characteristics of survey participants}

Table 1 summarizes baseline characteristics of the survey participants. A majority of the sample was composed of male participants (67.73\%). Participants were mostly 18 years old $(\mathrm{SD}=0.86)$, as they had just graduated from high school and entered Tsinghua University. The mean BMI was $21.21 \mathrm{~kg} / \mathrm{m}^{2}(\mathrm{SD}=3.80)$. A rather small 
Table 1 Baseline characteristics of survey participants

\begin{tabular}{|c|c|c|c|c|}
\hline & Total & Male & Female & $p$ \\
\hline N & 3097 & 2070 & 1027 & \\
\hline Age (yr), mean (SD) & $18.18(.85)$ & $18.22(.89)$ & $18.10(.76)$ & 0.000 \\
\hline \multicolumn{5}{|l|}{ Ethnicity, n (\%) } \\
\hline Han & $2891(90.43)$ & $1953(90.79)$ & $938(89.67)$ & \multirow[t]{2}{*}{0.313} \\
\hline Minority & $306(9.57)$ & $198(9.21)$ & $108(10.33)$ & \\
\hline \multicolumn{5}{|l|}{ Body mass index, mean (SD) } \\
\hline BMI $\left(\mathrm{kg} / \mathrm{m}^{2}\right)$ & $21.21(3.80)$ & $21.82(4.07)$ & $19.98(2.80)$ & 0.000 \\
\hline \multicolumn{5}{|l|}{ Smoking, $n(\%)$} \\
\hline Current smoker & $24(0.78)$ & $21(1.01)$ & $3(0.30)$ & \multirow[t]{2}{*}{0.038} \\
\hline Current nonsmoker & $3056(99.22)$ & $2065(98.99)$ & $991(99.70)$ & \\
\hline \multicolumn{5}{|l|}{ Drinking $n$ (\%) } \\
\hline Current drinker & $88(2.86)$ & $73(3.50)$ & $15(1.51)$ & \multirow[t]{2}{*}{0.002} \\
\hline Current nondrinker & $2992(97.14)$ & $2013(96.50)$ & $979(98.49)$ & \\
\hline \multicolumn{5}{|l|}{ Self-rated physical health, mean (SD) } \\
\hline Physical health score (1-10) & $5.78(1.83)$ & $5.77(1.86)$ & $5.80(1.75)$ & 0.687 \\
\hline \multicolumn{5}{|l|}{ Self-rated mental health, mean (SD) } \\
\hline Mental health score (1-10) & $7.07(1.89)$ & $7.09(1.92)$ & $7.02(1.81)$ & 0.339 \\
\hline \multicolumn{5}{|l|}{ Leisure-time vigorous physical activity, mean (SD) } \\
\hline Total minutes of vigorous physical activity in last week & $96.01(112.52)$ & $107.35(115.65)$ & $72.83(102.04)$ & 0.000 \\
\hline \multicolumn{5}{|l|}{ Leisure-time moderate physical activity, mean (SD) } \\
\hline Total minutes of moderate physical activity in last week & $212.12(173.76)$ & $219.63(174.92)$ & $197.52(170.63)$ & 0.001 \\
\hline \multicolumn{5}{|l|}{ Leisure-time MVPA, mean (SD) } \\
\hline Total minutes of vigorous and moderate physical activity in last week & $306.53(228.99)$ & $325.77(231.13)$ & $268.33(219.89)$ & 0.000 \\
\hline \multicolumn{5}{|l|}{ Walking, mean (SD) } \\
\hline Total minutes of walking in last week & $71.61(115.84)$ & $71.49(110.14)$ & $71.85(126.42)$ & 0.939 \\
\hline \multicolumn{5}{|l|}{ Sedentary behavior, mean (SD) } \\
\hline Total hours of sedentary behavior per day & $8.96(2.78)$ & $8.87(2.80)$ & $9.12(2.75)$ & 0.019 \\
\hline \multicolumn{5}{|l|}{ Sleeping behavior, mean (SD) } \\
\hline Total hours of sleeping behavior per day & $7.68(0.85)$ & $7.69(0.84)$ & $7.65(0.87)$ & 0.228 \\
\hline Total MTEs in last week, mean (SD) & $1732.45(1372.42)$ & $1843.00(1371.02)$ & 1510.93 (1348.88) & 0.000 \\
\hline
\end{tabular}

proportion of these freshmen were current smokers $(0.78 \%)$ and drinkers $(2.86 \%)$. Self-rated physical and mental health scores averaged 5.78 $(\mathrm{SD}=1.82)$ and $7.06(\mathrm{SD}=1.89)$, respectively. Survey participants on average engaged in 90.01 (SD =112.52) minutes of vigorous physical activity, $212.12(\mathrm{SD}=173.76)$ minutes of moderate physical activity, $306.53(\mathrm{SD}=228.99)$ minutes of MVPA, $71.61(\mathrm{SD}=115.84)$ minutes of walking, $1723.45(\mathrm{SD}=1372.42)$ total PA METminute scores, $8.94(\mathrm{SD}=2.78)$ hours per day of sedentary behavior and 7.68 ( $\mathrm{SD}=0.83$ ) hours per day of sleeping in the last week, respectively.

\section{The relationship between air pollution and PA}

Table 2 shows the mean variations of environmental measures and physical activity of the freshmen in the four follow-up surveys. As shown in Table 2, $\mathrm{PM}_{2.5}$ mean measures results had significantly increased from 72.90 to $165.13 \mu \mathrm{g} / \mathrm{m}^{3}$ in Beijing over the last seven days before the surveys being administered $(p<.001)$, and total minutes of MVPA in one week decreasing from 289.75 to 229.83 ( $\mathrm{p}<.001$ ). Figs $2-3$ shows the variations of air pollution and MVPA in the four follow-up surveys by male and female. It demonstrates there were significant declining outcomes in MVPA with the ambient $\mathrm{PM}_{2.5}$ air pollution, increasing among the freshmen over the follow-up period. There were also large variations in the percentage of rainy days and large variations in daytime temperature and average wind speed across the survey periods.

\section{Impact of air pollution on PA}

Table 3 reports the estimated effects of $\mathrm{PM}_{2.5}$ concentrations on individual-level outcomes pertaining to physical 
Table 2 Average physical activity, air pollution concentrations and other environmental variables in the last seven days before survey

\begin{tabular}{|c|c|c|c|c|c|c|c|c|}
\hline Survey order & 1nd & & $2 \mathrm{rd}$ & & $3 r d$ & & 4 th & \\
\hline \multicolumn{9}{|l|}{ Dependent variable } \\
\hline Vigorous PA (weekly minutes), mean (SD) & 95.81 & $(112.16)^{* *}$ & 104.76 & $(90.62)^{* *}$ & 77.18 & $(97.50)^{* * *}$ & 126.90 & $(104.29)^{* * *}$ \\
\hline Moderate PA (weekly minutes), mean (SD) & 213.46 & $(175.24)^{* *}$ & 195.64 & $(147.34)^{* * *}$ & 157.94 & $(131.37)^{* * *}$ & 177.35 & $(128.34)^{* * *}$ \\
\hline MVPA (weekly minutes), mean (SD) & 289.75 & $(226.77)$ & 286.96 & $(194.32)$ & 229.83 & $(183.69)^{* * *}$ & 300.55 & $(188.60)$ \\
\hline Walk PA (weekly minutes), mean (SD) & 71.91 & $(115.73)$ & 69.64 & $(112.51)$ & 73.30 & $(102.43)^{*}$ & 81.05 & $(108.85)^{* *}$ \\
\hline Sedentary behavior (hour/day), mean (SD) & 8.96 & $(2.78)^{* * *}$ & 9.43 & $(2.91)^{* *}$ & 9.21 & $(2.85)^{*}$ & 9.21 & $(2.92)^{*}$ \\
\hline Sleeping behavior (hour/day), mean (SD) & 7.68 & $(0.85)$ & 7.66 & $(0.91)$ & 7.87 & $(0.86)^{* * *}$ & 7.79 & $(0.99)^{* * *}$ \\
\hline Total Met, mean (SD) & 1835.67 & $(1381.20)$ & 1848.72 & (1221.28) & 1501.97 & $(1151.37)^{* * *}$ & 1994.27 & $(1228.22)^{* * *}$ \\
\hline \multicolumn{9}{|l|}{ Independent variables } \\
\hline PM2.5 $\left(\mu \mathrm{g} / \mathrm{m}^{3}\right)$, mean (SD) & 72.90 & $(50.26)$ & 68.78 & $(58.48)$ & 165.13 & $(131.98)^{* * *}$ & 92.88 & $(70.43)^{*}$ \\
\hline \multicolumn{9}{|l|}{ Covariates variables } \\
\hline Temperature $\left({ }^{\circ} \mathrm{C}\right)$, mean $(\mathrm{SD})$ & 25.36 & $(2.87)$ & 11.64 & $(4.20)^{* * *}$ & 12.17 & $(5.13)^{* * *}$ & 28.73 & $(3.00)$ \\
\hline Wind $(m / s)$, mean $(S D)$ & 3.23 & $(0.75)$ & 3.55 & $(0.65)$ & 3.38 & $(1.00)$ & 3.41 & $(0.44)$ \\
\hline Rain \%, mean (SD) & 0.36 & $(0.51)$ & 0.18 & $(0.41)$ & 0.08 & $(0.29)$ & 0.18 & $(0.41)$ \\
\hline
\end{tabular}

Results of repeated measures one-way ANOVA analysis. ${ }^{*} P<0.05 ;{ }^{* *} P<0.01 ;{ }^{* * *} P<0.001$

activity level using linear individual fixed-effect regressions. $\mathrm{PM}_{2.5}$ concentrations were found to be negatively associated with total minutes of physical activity in the last week among survey participants. Specifically, an increase in $\mathrm{PM}_{2.5}$ concentrations by one standard deviation (i.e., $44.72 \mu \mathrm{g} / \mathrm{m}^{3}$ ) was associated with a reduction in weekly total minutes of vigorous physical activity by 22.32 (95\% confidence interval $[\mathrm{CI}]=-24.88,-19.77)$, a reduction in weekly total minutes of moderate physical activity by $10.63(95 \% \mathrm{CI}=-14.61,-6.64)$, a reduction in weekly total minutes of MVPA by 32.45 (95\% $\mathrm{CI}=-37.63,-27.28)$ and a reduction in weekly total physical activity MET-minute scores by 226.14 (95\% $\mathrm{CI}=-256.06,-196.21)$. Ambient $\mathrm{PM}_{2.5}$ concentration was not associated with minutes spent walking by the freshmen $(p>0.05)$.

The estimated effects were similar between the male and female students except for weekly total minutes of

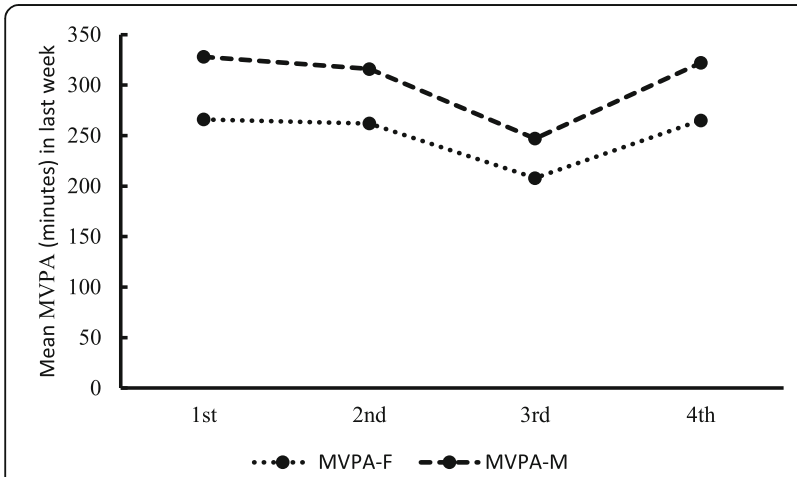

Fig. 2 Mean of ambient $P M_{2.5}$ air pollution $\left(\mu \mathrm{g} / \mathrm{m}^{3}\right)$ in last week of the survey during the period of the four times follow-up moderate physical activity. An increase in ambient $\mathrm{PM}_{2.5}$ concentration by one standard deviation was associated with a statistically significant reduction in weekly total minutes of moderate physical activity by 13.27 (95\% $\mathrm{CI}=-18.24,-8.29)$ among males $(p<.001)$; whereas the estimated decline by $4.79(95 \% \mathrm{CI}=-11.41,-1.83)$ in weekly total minutes of moderate physical activity in response to an elevated $\mathrm{PM}_{2.5}$ concentration level was much smaller and statistically nonsignificant for females ( $\mathrm{p}>0.05)$.

Regarding other environmental measures and individual-level covariates (results not shown in table), wind speed and percentage of rainy days over the last seven days were consistently negatively associated with physical activity, but positively associated with daily average daytime temperature. High self-rated physical

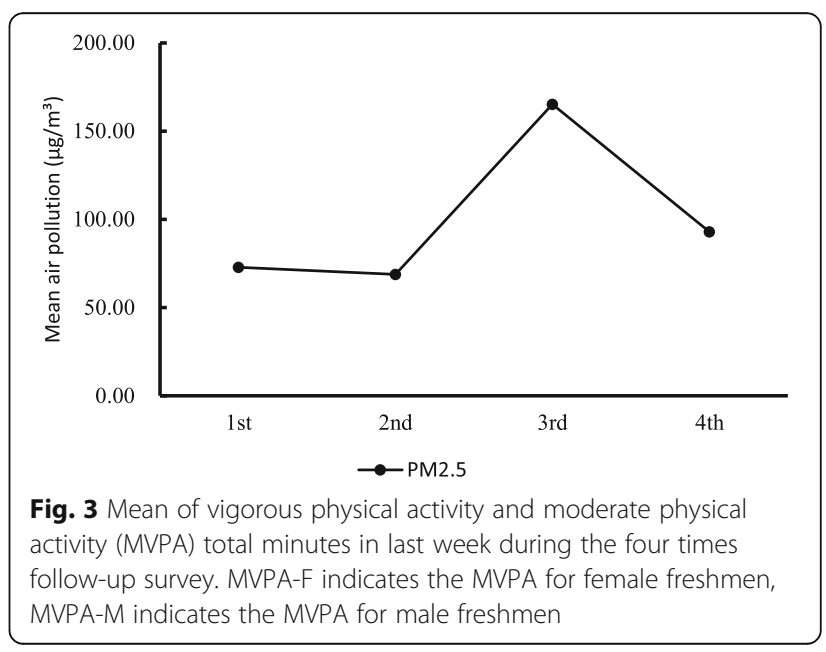


Table 3 Estimated effects of $\mathrm{PM}_{2.5}$ on individual-level physical activity outcomes by sex

\begin{tabular}{|c|c|c|c|c|c|c|}
\hline \multirow[t]{2}{*}{ Dependent variable } & \multicolumn{2}{|l|}{ Total } & \multicolumn{2}{|l|}{ Male only } & \multicolumn{2}{|l|}{ Female only } \\
\hline & $\begin{array}{l}\text { Coefficient } \\
(95 \% \mathrm{Cl})\end{array}$ & $\begin{array}{l}\text { \# Observations } \\
\text { (\# participants) }\end{array}$ & $\begin{array}{l}\text { Coefficient } \\
(95 \% \mathrm{Cl})\end{array}$ & $\begin{array}{l}\text { \# Observations } \\
\text { (\# participants) }\end{array}$ & $\begin{array}{l}\text { Coefficient } \\
(95 \% \mathrm{Cl})\end{array}$ & $\begin{array}{l}\text { \# Observations } \\
\text { (\# participants) }\end{array}$ \\
\hline $\begin{array}{l}\text { Total minutes of vigorous physical } \\
\text { activity in last week }\end{array}$ & $\begin{array}{l}-22.32^{* * *} \\
(-24.88,-19.77)\end{array}$ & $11,318(3241)$ & $\begin{array}{l}-21.70^{* * *} \\
(-24.94,-18.46)\end{array}$ & 7654 (2189) & $\begin{array}{l}-23.55^{* * * *} \\
(-27.59,-19.52)\end{array}$ & $3664(1065)$ \\
\hline $\begin{array}{l}\text { Total minutes of moderate physical } \\
\text { activity in last week }\end{array}$ & $\begin{array}{l}-10.63^{* * *} \\
(-14.61,-6.64)\end{array}$ & $11,045(3231)$ & $\begin{array}{l}-13.27^{* * *} \\
(-18.24,-8.29)\end{array}$ & $7416(2181)$ & $\begin{array}{l}-4.79 \\
(-11.41,1.83)\end{array}$ & $3629(1063)$ \\
\hline Total minutes of MVPA & $\begin{array}{l}-32.45^{* * *} \\
(-37.63,-27.28)\end{array}$ & $10,832(3228)$ & $\begin{array}{l}-34.72^{* * *} \\
(-41.22,-28.23)\end{array}$ & 7297 (2179) & $\begin{array}{l}-27.29^{* * *} \\
(-35.75,-18.83)\end{array}$ & $3535(1062)$ \\
\hline Total minutes of walk in last week & $\begin{array}{l}-.86 \\
(-3.84,2.12)\end{array}$ & $11,018(3233)$ & $\begin{array}{l}-2.68 \\
(-6.32, .95)\end{array}$ & 7411 (2183) & $\begin{array}{l}3.30 \\
(-1.91,8.52)\end{array}$ & $3607(1063)$ \\
\hline Total met in last week & $\begin{array}{l}-226.14^{* * *} \\
(-256.06,-196.21)\end{array}$ & $11,589(3242)$ & $\begin{array}{l}-238.10^{* * *} \\
(-275.72,-200.47)\end{array}$ & 7809 (2190) & $\begin{array}{l}-198.19^{* * *} \\
(-246.91,-149.46)\end{array}$ & $3780(1065)$ \\
\hline
\end{tabular}

Separate individual fixed-effect regressions were performed to estimate the effects of air pollution concentrations on samples stratified by sex

Models adjust for all time-variant individual characteristics listed in Table 1 (i.e., age, BMI, smoking status, drinking status, self-rated physical health, and self-rated mental health) and environmental variables listed in Table 2 (average daily temperature, average wind speed, and percentage of rainy day in last week). ${ }^{*} P<0.05$; ${ }^{* *} P<0.01 ;{ }^{* * *} P<0.001$

health was positively associated with physical activity and high self-rated mental health was positively associated with physical activity over in the past week.

\section{Discussion}

This study assessed the longitudinal relationship linking ambient $\mathrm{PM}_{2.5}$ concentration to behavioral modifications pertaining to weekly physical activity level among university freshmen in Beijing from 2012 to 2013. Ambient $\mathrm{PM}_{2.5}$ concentration was found to be negatively associated with weekly total minutes of physical activity behavior among survey participants. The impact of ambient $\mathrm{PM}_{2.5}$ concentration on weekly total minutes of moderate physical activity tended to be greater among males than females. To our knowledge, this study is the first known cohort study to examine the impact of air pollution concentration on young adults' health behaviors in a middle-income country. This study reveals that air pollution leads to a decrease in MVPA behavior in young adults in China.

In our study, ambient $\mathrm{PM}_{2.5}$ was negatively associated with physical activity in Chinese youth. Our results suggest an increase in ambient $\mathrm{PM}_{2.5}$ dismisses physical activity among freshmen. This finding is consisted with previous research $[21,25,26]$ assessing the relationship between air pollution and physical inactivity, which finds that an increase $\mathrm{PM}_{2.5}$ leads to the increasing prevalence of physical inactivity. These three studies all found that air pollution may negatively influence physical activity behavior; moreover, the air pollution problem in China has attracted worldwide attention in recent years. Previous studies have reported that air pollution, especially ambient air pollution, dismiss outdoor physical activity among children and adolescents in China [36, 37], which is a distinctive public health challenge in China [38]. In our study, we found that there was a significantly declining impact of ambient $\mathrm{PM}_{2.5}$ on vigorous physical activity, which is inconsistent with previous results [39]. A recent study in Beijing of 40 Han Chinese participants found that there was no impact of ambient $\mathrm{PM}_{2.5}$ and ambient temperature on physical activity [39]. A possible explanation for this difference could be that 40 participants is too small of a sample and may have been subjected to social behavior desirability bias [40].

The WHO guideline recommends that adults need a minimum of 150 min of moderate-to-vigorous physical activity (MVPA) per day [41]. Our finding shows that an increase in $\mathrm{PM}_{2.5}$ concentration significantly discourages Chinese freshmen students from performing more than 30 min of MVPA behavior per week, less than $20 \%$ of the current guideline. MVPA is considered as an effective intervention to promote health in youth [42]. Previous studies show that MVPA can help prevent weight gain and maintain a healthy body weight in youth [43]; however, physical inactivity and sedentary behavior are growing problems among Chinese youth [44]. The prevalence of obesity among Chinese youth more than doubled, from 1993 (6.1\%) to 2009 (13.1\%) [45]. According to the China Health and Nutrition Survey, the most common forms of MVPA among Chinese youth are performed outdoors [46]. Exposure to air pollution and outdoor physical activity among Chinese youth has become a serious public health problem [36]. Our result provides the new evidence that air pollution dismisses Chinese youth from performing MVPA behavior, thus exposing them to elevated health risks.

Previous studies $[47,48]$ showed that other weatherrelated variable conditions, such as temperature, strong wind, and rain, may also play important roles in influencing physical activity levels. This finding was consistent with several previous publications that found that wind speed and percentage of rainy days were consistently 
negatively associated with physical activity, but positively associated with daily average daytime temperature. For example, individuals may decrease MVPA when temperature is too cold. In the winter in Beijing, it was much colder (average $12{ }^{\circ} \mathrm{C}$ ) than in the summer (average $25^{\circ} \mathrm{C}$ ). At the same time, the air pollution was much worse in the winter than in the summer [49].

Our finding indicates significant reductions in physical activity relative to ambient $\mathrm{PM}_{2.5}$ level. In our study analysis, a change in ambient $\mathrm{PM}_{2.5}$ concentration by one standard deviation $\left(44.72 \mu \mathrm{g} / \mathrm{m}^{3}\right)$ is similar to the government air quality one cut off standard $\left(50 \mu \mathrm{g} / \mathrm{m}^{3}\right)$ threshold. There are six thresholds for air pollution in China [2]: 0-50 $\mu \mathrm{g} / \mathrm{m}^{3}$ (good, green warning), 51-100 $\mu \mathrm{g} /$ $\mathrm{m}^{3}$ (fair, yellow warning), $101-150 \mu \mathrm{g} / \mathrm{m}^{3}$ (mild pollution, orange warning), 151-200 (moderate pollution, red warning), $201-300 \mu \mathrm{g} / \mathrm{m}^{3}$ (severe pollution, purple warning) and $>300 \mu \mathrm{g} / \mathrm{m}^{3}$ (serious pollution, maroon warning). It is interesting to note that in our study, ambient $\mathrm{PM}_{2.5}$ was in the yellow warning level during the first, second and forth follow-up survey, the freshmen's physical activity level did not decrease in a linearly regression with an increase in $\mathrm{PM}_{2.5}$ value. When ambient $\mathrm{PM}_{2.5}$ was in the red warning level during the third follow-up period, participants' physical activity decreased rapidly. A potential explanation for the difference in air pollution level contributors in to decreasing physical activity is that smog air, warning guidelines, and media alerts create a combined effect in dismissing people from performing physical activity, especially in outdoor exercise and vigorous physical activity. Consistent with previous research, media alert perceptions of air pollution were statistically significantly associated with physical activity behavior in adults [22].

Subgroup analyses suggested that male's moderate physical activity patterns tended to be more responsive to the variations in ambient $\mathrm{PM}_{2.5}$ concentration than female's. This could be partially explained by the Chinese gender perception difference of bad air quality risk. Compared to males, females tend to perceive poor air quality as a health risk and may reduce their risk of exposure by changing or reducing their outdoor activity. However, our findings on the sex differences among youth are rather preliminary and warrant replication by future studies.

The strengths of this study lie in its longitudinal study design, reliable and time-sensitive environmental measures and a comprehensive sample of 4 waves of follow-up for all freshmen at Tsinghua University from 2012 to 2013. Nevertheless, a few major limitations of this study should be noted. The sample was from only one university. Freshmen cohorts from one university are unlikely to represent the entire undergraduate population in Beijing, or nationwide, which confines the generalizability of the study findings. Future follow-up studies with representative samples are warranted to replicate findings of this study and produce generalizable estimates. Health behaviors were all self-reported and subject to recall error and social desirability bias [50]. The Mission China Air Quality Monitoring Program led by the Ministry of Environmental Protection of the People's Republic of China had data air pollution dating back to December 2013, but did not provide any data earlier than this time. The U.S. Department of State published data on air pollution in 2011 but only had ambient $\mathrm{PM}_{2.5}$ data. Beijing covers a rather large metropolitan area and different neighborhoods may have different levels of air pollution concentration. Using the city-average air pollution measure could have masked considerable local variations. The individual fixed-effect models eliminated confounding bias from factors that remained constant within-participant over time, but it did not control for more transient unobserved factors such as daily variations in pains and emotions. Moreover, it is the increase in potential error by conducting multiple regression analyses with highly correlated dependent variables. Previous studies have shown that there is a declining trend of physical activity behavior among freshmen university students as they progress through their first academic year, even without exposure to air pollution [51]. Therefore, this is a significant confounder, and future follow-up studies should control the confounder and figure out a clearer relationship between air pollution and physical activity behavior among freshmen university students. Additional limitations of the present study include the following: it is not known if individuals are exercising indoors or outdoors, changes in physical activity participation may not vary by air pollution if an individual is active indoors, heavy air pollution has gradually become a norm that residents in many large Chinese cities have to live with, and switching from outdoor physical activities (eg, running on sidewalks) to indoor exercises (eg, running on a treadmill) could partially offset the disruption of air pollution.

\section{Conclusion}

This study examined the longitudinal relationship between air pollution concentration and behavioral modifications pertaining to weekly physical activity level among university freshmen in Beijing, China. Ambient air pollution concentration was negatively associated with weekly total minutes of physical activity behavior. Ambient $\mathrm{PM}_{2.5}$ plays a key role in discouraging youth from performing physical activity behavior. Future studies are warranted to replicate findings of this study in other Chinese cities and universities, and policy interventions are urgently called to reduce air pollution level in China. 


\section{Abbreviations}

BMI: Body mass index; MET: Metabolic equivalent; MVPA: Moderate-tovigorous physical activity; PM2.5: Particulate matter with a diameter less than $2.5 \mu \mathrm{m}$

\section{Acknowledgements}

We are grateful for the support of all of the participants in the study. We are grateful to the U.S. Department of State for providing the hourly $\mathrm{PM}_{25}$ data and to the China Meteorological Administration for providing the average daytime temperature $(C)$, average wind speed $(\mathrm{m} / \mathrm{s})$ and percentage of rainy days data in Beijing, China.

\section{Funding}

This study was supported by the National Social Science Foundation of China (17CTY020 and 13BTY049) and by the Tsinghua University Initiative Scientific Research Program (20151080433)

\section{Availability of data and materials}

The datasets generated and/or analyzed during the current study are not publicly available due to confidentially reasons, but are available from the corresponding author on reasonable request.

\section{Authors' contributions}

HY designed and conceived the study. MY and RZ were involved in the collection of air pollution and ambient temperature data. SPG contributed to the writing of the manuscript. HY analyzed the data and wrote the manuscript. All authors read and approved the final manuscript.

\section{Ethics approval and consent to participate}

This study was approved by the Tsinghua University Institutional Review Board (IRB \#2012534001). All participants gave written informed consent during the survey.

\section{Consent for publication}

Not applicable.

\section{Competing interests}

The authors declare that they have no competing interests.

\section{Publisher's Note}

Springer Nature remains neutral with regard to jurisdictional claims in published maps and institutional affiliations.

\section{Author details}

'Department of Physical Education, Tsinghua University, Tsinghua Yuan Str, Beijing 100084, China. ${ }^{2}$ Department of Reference Service in Library, Renmin University of China, Zhongguan Cun Str, Beijing 100872, China. ${ }^{3}$ Department of Interdisciplinary Health Sciences, University of Illinois at Urbana-Champaign, Urbana, IL, USA.

Received: 13 July 2017 Accepted: 27 September 2017

Published online: 05 October 2017

\section{References}

1. Prüss-Üstün A, Corvalán C. Preventing disease through healthy environments. Towards an estimate of the environmental burden of disease. Geneva: World Health Organization. 2006;

2. Guan WJ, Zheng XY, Chung KF, Zhong NS. Impact of air pollution on the burden of chronic respiratory diseases in China: time for urgent action. Lancet. 2016;388:1939-51.

3. Xing YF, YH X, Shi MH, Lian YX. The impact of PM2.5 on the human respiratory system. J Thorac Dis. 2016;8:E69-74.

4. Auchincloss AH, Diez Roux AV, Dvonch JT, Brown PL, Barr RG, Daviglus ML, Goff DC, Kaufman JD, O'Neill MS. Associations between recent exposure to ambient fine particulate matter and blood pressure in the Multi-ethnic Study of Atherosclerosis (MESA). Environ Health Perspect. 2008;116:486-91.

5. Brook RD, Rajagopalan S, Pope CA, 3rd, Brook JR, Bhatnagar A, Diez-Roux AV, Holguin F, Hong Y, Luepker RV, Mittleman MA, et al. Particulate matte air pollution and cardiovascular disease: An update to the scientific statement from the American Heart Association. Circulation2010;121: 2331-2378
6. Cakmak S, Dales R, Leech J, Liu L. The influence of air pollution on cardiovascular and pulmonary function and exercise capacity: Canadian Health Measures Survey (CHMS). Environ Res. 2011;111:1309-12.

7. Miller KA, Siscovick DS, Sheppard L, Shepherd K, Sullivan JH, Anderson GL, Kaufman JD. Long-term exposure to air pollution and incidence of cardiovascular events in women. N Engl J Med. 2007:356:447-58.

8. World Health Organization. Physical activity. 2017. http://www.who.int/ topics/physical_activity/en/ Accessed 28 Mar 2017.

9. Warburton DE, Nicol CW, Bredin SS. Health benefits of physical activity: the evidence. Can Med Assoc J. 2006;174:801-9.

10. World Health Organization. Global recommendations on physical activity for health. 2010. http://www.who.int/dietphysicalactivity/factsheet_ recommendations/en/ Accessed Jun 242017.

11. Machado-Rodrigues AM, Leite N, Coelho e Silva MJ, Valente-dos-Santos J, Martins RA, Mascarenhas LP, Boguszewski MC, Padez C, Malina RM. Relationship between metabolic syndrome and moderate-to-vigorous physical activity in youth. J Phys Act Health. 2015:12:13-9.

12. Lee IM, Shiroma EJ, Lobelo F, Puska P, Blair SN, Katzmarzyk PT. Effect of physical inactivity on major non-communicable diseases worldwide: an analysis of burden of disease and life expectancy. Lancet. 2012;380:219-29.

13. Hallal PC, Andersen LB, Bull FC, Guthold R, Haskell W, Ekelund U. Global physical activity levels: surveillance progress, pitfalls, and prospects. Lancet. 2012;380:247-57.

14. Committee PAGA. Physical activity guidelines advisory committee report, 2008. Washington, DC: US Department of Health and Human Services. 2008; 2008:A1-H14.

15. Garber CE, Blissmer B, Deschenes MR, Franklin BA, Lamonte MJ, Lee IM, Nieman DC, Swain DP. American College of Sports Medicine position stand. Quantity and quality of exercise for developing and maintaining cardiorespiratory, musculoskeletal, and neuromotor fitness in apparently healthy adults: guidance for prescribing exercise. Med Sci Sports Exerc. 2011:43:1334-59.

16. Muntner P, Gu D, Wildman RP, Chen J, Qan W, Whelton PK, He J. Prevalence of physical activity among Chinese adults: results from the International Collaborative Study of Cardiovascular Disease in Asia. Am J Public Health. 2005;95:1631-6.

17. Colley RC, Garriguet D, Janssen I, Craig CL, Clarke J, Tremblay MS. Physical activity of Canadian children and youth: accelerometer results from the 2007 to 2009 Canadian Health Measures Survey. Health Rep. 2011;22:15-23.

18. Sothern MS, Loftin M, Suskind RM, Udall JN, Blecker U. The health benefits of physical activity in children and adolescents: implications for chronic disease prevention. Eur J Pediatr. 1999;158:271-4.

19. Dales $R$, Chen $L$, Frescura AM, Liu L, Villeneuve PJ. Acute effects of outdoor air pollution on forced expiratory volume in $1 \mathrm{~s}$ : a panel study of schoolchildren with asthma. Eur Respir J. 2009:34:316-23.

20. Rundell KW, Caviston R. Ultrafine and fine particulate matter inhalation decreases exercise performance in healthy subjects. J Strength Cond Res. 2008;22:2-5

21. Roberts JD, Voss JD, Knight B. The association of ambient air pollution and physical inactivity in the United States. PLoS One. 2014;9:e90143.

22. Wen XJ, Balluz L, Mokdad A. Association between media alerts of air quality index and change of outdoor activity among adult asthma in six states, BRFSS, 2005. J Community Health. 2009:34:40-6.

23. Cutrufello PT, Rundell KW, Smoliga JM, Stylianides GA. Inhaled whole exhaust and its effect on exercise performance and vascular function. Inhal Toxicol. 2011;23:658-67.

24. Marr LC, Ely MR. Effect of air pollution on marathon running performance. Med Sci Sports Exerc. 2010;42:585-91.

25. Wen XJ, Balluz LS, Shire JD, Mokdad AH, Kohl HW. Association of selfreported leisure-time physical inactivity with particulate matter 2.5 air pollution. J Environ Health. 2009;72:40-4. quiz 45

26. An $\mathrm{R}$, Xiang X. Ambient fine particulate matter air pollution and leisure-time physical inactivity among US adults. Public Health. 2015;129:1637-44.

27. Hongjun Y, Ruopeng A, Andrade F. Ambient Fine Particulate Matter Air Pollution and Physical Activity : A Longitudinal Study of University Retirees in Beijing, China. Am J Health Behav. 2017:41:401-10.

28. United States Environmental Protection Agency. EPA announces next round of clean air standards to reduce harmful soot pollution. 2017. http:// yosemite.epa.gov/opa/admpress.nsf/d0cf6618525a9efb85257359003fb69d/ a7446ca9e228622b85257ad400644d82!OpenDocument Accessed January 29 2017 
29. Yang G, Wang Y, Zeng Y, Gao GF, Liang X, Zhou M, Wan X, Yu S, Jiang Y, Naghavi M, et al. Rapid health transition in China, 1990-2010: findings from the Global Burden of Disease Study 2010. Lancet. 2013;381:1987-2015.

30. Yu H, An R, Andrade F. Ambient Fine Particulate Matter Air Pollution and Physical Activity: A Longitudinal Study of University Retirees in Beijing, China. Am J Health Behav. 2017:41:401-10.

31. Lee PH, Macfarlane DJ, Lam T, Stewart SM. Validity of the international physical activity questionnaire short form (IPAQ-SF): A systematic review. Int J Behav Nutr Phys Act. 2011;8:115.

32. Bassett DR, Jr. International physical activity questionnaire: 12-country reliability and validity. Med Sci Sports Exerc2003;35:1396.

33. Macfarlane DJ, Lee CC, Ho EY, Chan KL, Chan DT. Reliability and validity of the Chinese version of IPAQ (short, last 7 days). J Sci Med Sport. 2007:10:45-51.

34. Craig CL, Marshall AL, Sjostrom M, Bauman AE, Booth ML, Ainsworth BE, Pratt M, Ekelund U, Yngve A, Sallis JF, Oja P. International physical activity questionnaire: 12-country reliability and validity. Med Sci Sports Exerc. 2003; 35:1381-95.

35. Committee IR: Guidelines for data processing and analysis of the International Physical Activity Questionnaire (IPAQ)-short and long forms. 2005.

36. Si Q, Cardinal BJ. The Health Impact of Air Pollution and Outdoor Physical Activity on Children and Adolescents in Mainland China. J Pediatr. 2017;180: $251-5$.

37. Li F, Liu Y, Lu J, Liang L, Harmer P. Ambient air pollution in China poses a multifaceted health threat to outdoor physical activity. J Epidemiol Community Health. 2015;69:201-4.

38. Lu J, Liang L, Feng Y, Li R, Liu Y. Air Pollution Exposure and Physical Activity in China: Current Knowledge, Public Health Implications, and Future Research Needs. Int J Environ Res Public Health. 2015;12:14887-97.

39. Wang G, Li B, Zhang X, Niu C, Li J, Li L, Speakman JR. No seasonal variation in physical activity of Han Chinese living in Beijing. Int J Behav Nutr Phys Act. 2017;14:48.

40. Button KS, loannidis JP, Mokrysz C, Nosek BA, Flint J, Robinson ES, Munafò MR. Power failure: why small sample size undermines the reliability of neuroscience. Nat Rev Neurosci. 2013;14:365-76.

41. Centers for Disease Control and Prevention. 2008 physical activity guidelines for Americans. 2008. https://www.cdc.gov/physicalactivity/downloads/pa_ fact_sheet_adults.pdf. Accessed January 292017.

42. Nader PR, Bradley RH, Houts RM, McRitchie SL, O'Brien M. Moderate-tovigorous physical activity from ages 9 to 15 years. JAMA. 2008;300:295-305.

43. Mitchell JA, Pate RR, Espana-Romero V, O'Neill JR, Dowda M, Nader PR. Moderate-to-vigorous physical activity is associated with decreases in body mass index from ages 9 to 15 years. Obesity (Silver Spring). 2013;21:E280-93.

44. Yu Z, Han S, Chu J, Xu Z, Zhu C, Guo X. Trends in overweight and obesity among children and adolescents in China from 1981 to 2010: a metaanalysis. PLoS One. 2012;7:e51949

45. Zong XN, Li H. Physical growth of children and adolescents in China over the past 35 years. Bull World Health Organ. 2014;92:555-64.

46. Tudor-Locke C, Ainsworth BE, Adair LS, Du S, Popkin BM. Physical activity and inactivity in Chinese school-aged youth: the China Health and Nutrition Survey. Int J Obes Relat Metab Disord. 2003:27:1093-9.

47. Hamilton SL, Clemes SA, Griffiths PLUK. adults exhibit higher step counts in summer compared to winter months. Ann Hum Biol. 2008:35:154-69.

48. Ridgers ND, Salmon J, Timperio A. Too hot to move? Objectively assessed seasonal changes in Australian children's physical activity. Int J Behav Nutr Phys Act. 2015;12:77.

49. Song C, Pei T, Yao L. Analysis of the characteristics and evolution modes of PM2.5 pollution episodes in Beijing, China during 2013. Int J Environ Res Public Health. 2015;12:1099-111.

50. Althubaiti A. Information bias in health research: definition, pitfalls, and adjustment methods. J Multidiscip Healthc. 2016;9:211-7.

51. Deforche B, Van Dyck D, Deliens T, De Bourdeaudhuij I. Changes in weight, physical activity, sedentary behaviour and dietary intake during the transition to higher education: a prospective study. Int J Behav Nutr Phys Act. 2015;12:16.

\section{Submit your next manuscript to BioMed Central and we will help you at every step:}

- We accept pre-submission inquiries

- Our selector tool helps you to find the most relevant journal

- We provide round the clock customer support

- Convenient online submission

- Thorough peer review

- Inclusion in PubMed and all major indexing services

- Maximum visibility for your research

Submit your manuscript at www.biomedcentral.com/submit

CBiomed Central 